\title{
Route Optimization Technique to Support Multicast in Mobile Networks*
}

\author{
Kiyong Park ${ }^{1}$, Sunyong Han ${ }^{1}$, Bok-gyu Joo ${ }^{2}$, Keecheon Kim ${ }^{1}$, and Jinpyo Hong ${ }^{3}$ \\ Kon-kuk University, \\ ${ }^{2}$ Hongik University, \\ ${ }^{3}$ Hankuk University of Foreign Studies, \\ Seoul, Korea \\ kypark@cclab.konkuk.ac.kr, \{syhan,kckim\}@konkuk.ac.kr, \\ bkjoo@hongik.ac.kr, jphong@hufs.ac.kr
}

\begin{abstract}
The technology called Network Mobility (NEMO) is proposed recently by IETF to support the network mobility. Meanwhile, as the needs of group communications increase in the internet, supporting multicast services became a challenging issue on NEMO as on Mobile IP. This paper explores issues and problems of providing multicast service on mobile networks and presents a route optimization technique along with an architecture that enables optimal and efficient multicast service on NEMO environments.
\end{abstract}

\section{Introduction}

Since IETF proposed Mobile IP technology [1] as a technique to provide host mobility, many research works have been made to support host mobility in wireless environments. Nowadays, people want to enjoy surfing the internet even in the moving vehicles such as buses and planes, even if their equipments do not support mobility. Meanwhile, the importance of group communications has been recognized in various applications such as remote lectures, on-line games, etc. This is true even in wireless and mobile environments.

IETF recently proposed a new architecture called Network Mobility(NEMO) basic support architecture in order to answer the requirements for network mobility [2][3]. In early Mobile-IP(MIP) technologies, basic architectures such as MIP-BT, MIP-RS [7] and Multicast Proxy Server [8], were suggested to provide multicast service for mobile hosts. Because the NEMO basic support architecture is based on Mobile IP, it inherits from Mobile IP the same fundamental problems such as route optimization problem [9], tunnel convergence problem, mass of multicast problem, when it is used to support multicast for mobile networks. Therefore, we have to reinvestigate multicast support mechanism suitable for NEMO environments.

In this paper, we propose new schemes for supporting multicast on NEMO, by extending the functions of mobile router and adding new parts of router advertisement messages in MIPv6 network. We adopted Prefix Delegation mechanism suggested for route optimization in MIPv6 in order to provide route optimization on multicasting.

\footnotetext{
* This research is supported by University IT Research Center Project.
} 
For seamless multicast service even when a mobile network changes its location, we extended mobile router functions to manage the creation and coordination of dynamic tunneling with other multicast routers. Our approach provides an efficient and optimal multicasting and will become a fundamental architecture for supporting multicast on NEMO.

\section{Related Researches}

\subsection{Network Mobility (NEMO) Basic Support Architecture}

To support network mobility, some basic requirements are to be satisfied [4] on existing networks. Those requirements are session continuity, connectivity, reachability, and nested mobility support. Most of the requirements are met by employing a mobile router(MR), which is in charge of its own network mobility. Because the MR acts not only as a mobile node(MN) but also as a router, it provides a route path to on-link nodes in its network and guarantees network connectivity to the internet. To support those requirements basically in NEMO basic support architecture, bi-directional tunneling is necessary between the MR and its Home Agent (HA) so that on-link nodes in the MR's network can be reachable in the internet

A Correspondent Node $(\mathrm{CN})$ may not know where the target node is placed at the first time during communication after a mobile network finished its movement. Therefore, the HA must find out the location of the node and forward data to it. The location information of the node with which the $\mathrm{CN}$ wants to communicate, however, does not exist in the binding list of the HA. This is because the node didn't make any registration request message for movement notification. In spite of that, the HA can find out the location information of the MR containing the node, because the MR makes a registration request process to the HA when it moves. So the HA tunnels to MR's CoA and the MR forward the data from the HA to the nodes in its network.

\subsection{MIP-BT (Bi-directional Tunneling) and MIP-RS (Remote Subscription)}

In MIP-BT, a mobile node in a foreign network receives multicast data packets from the mobility agent in the home network by bi-directional tunneling. This approach assumes that the home agent has multicast router functions or there is a multicast router in the home network. The home agent intercepts multicast packets that the mobile node used to receiving, encapsulates and transmits them to the mobility agent in the foreign network (called 'foreign agent'). When the foreign agent receives these packets, it decapsulates them and sends to the local network. In MIP-RS, when a mobile node moves to a new network, it sends IGMP messages to the local network in order to rejoin the multicast group [6], so that it can receive multicast data packets from the local multicast router of the foreign network.

The major advantage of MIP-BT approach is it can minimize data packets loss and there is no delay caused by reconstructing a multicast route tree. The major advantage of MIP-RS approach is that the mobile node is served directly by the local multicast router, so that multicast data packet route is optimized 


\subsection{Prefix Delegation (PD) Mechanism for Route Optimization on NEMO}

NEMO basic support architecture preserves session continuity by employing bidirectional tunnel between a MR and its HA. This scheme is reasonable only for a small-scale mobile network because MR has to encapsulate and decapsulate all packets for its MNs.

The purpose of PD mechanism [5] is to enable MNs behind the MR to perform Mobile IPv6 route optimization. In this mechanism, every MNs under a mobile network is allocated native routable internet address as its CoA from the network prefixes in Route Advertisement (RA) messages. That means a MR does not advertise the home network prefixes but newly-acquired network prefixes from the active router (AR)'s RA message. This process is repeated recursively on nested mobile networks, and eventually all MNs and MRs under the AR can be native routable.

\subsection{Summary of Current Problems}

Because NEMO basic support architecture has inherited Mobile IP and related techniques, many serious problems in supporting multicast in Mobile IP still exist in NEMO. Major problems are tunnel convergence problem, mass of multicast problem, and route optimization problem. Furthermore, because NEMO basic support is using bi-directional tunnel, multicasting on NEMO has the serious weakness called the pinball route problem [10], which can be classified into a route optimization problem.

If MIP-BT is adopted on NEMO, the tunnel convergence problem occurs and it gives much overhead not only on MRs but also on AR because they must process many tunnels, while the problem gives load only to AR or mobility agent on Mobile IP. Furthermore, the more networks are nested, the more damages occur in the entire mobile network. If MIP-RS is adopted on NEMO, MNs in the mobile network can not join a multicast group, because they don't know where multicast routers are located. Moreover, their IGMP [6] messages cannot be routed to a multicast router, because their IGMP messages may not be routed by the MR's upper router for each different mobile network prefixes.

\section{Architecture}

\subsection{Basic Concepts}

Firstly, we must handle the multicast support on a mobile network itself, otherwise no MNs in mobile network are sure of the reception of multicast data. Furthermore, lots of tunnels will generate network congestions in the mobile network and its ascendants. This problem, called tunnel convergence and mass of multicast problem, cannot be solved until the mobile network has a multicast router or the MR has multicast router functions. Even though there is a multicast router in the mobile network or MR has multicast router functions, we still encounter another problem that the MR or multicast router cannot have multicast route tree statically with any fixed multicast router because of network mobility. 
For above reasons, we assume that in our architecture, multicast router functions are built in the MR, and the MR and the AR must be in charge of notifying default multicast router (DmR) information to its descendant MRs to create multicast route tree. This DmR Information is an internet address of a fixed multicast router in the internet. The information can be set manually for AR and dynamically in run time for MR.

In NEMO basic support architecture, bi-directional tunnel is used for a MR to communicate with its home network. But it causes a serious problem of route optimization, called the pinball route problem. It happens again in the case of multicasting whenever a mobile network is nested. To overcome this weakness, we adopt the Prefix Delegation mechanism. It achieves route optimization of tunnel between a MR and a multicast router. That is, multicast tunnels in our architecture are made from the internet address of multicast router directly to MR's CoA.

In addition, when a MR receives a RA message it must be able to distinguish the source of the message, whether it is from the AR or an upper MR. We use a specialized bit, named ' $M$ ', in the RA message. If the ' $M$ ' bit is checked, the RA message is from an upper MR and the network is nested. We designated for ' $M$ ' bit one of five reserved bits in RA message format.

\subsection{Operation Overview}

Fig. 1 shows how network components interact in NEMO for multicasting and how the tunnels are established to each router.

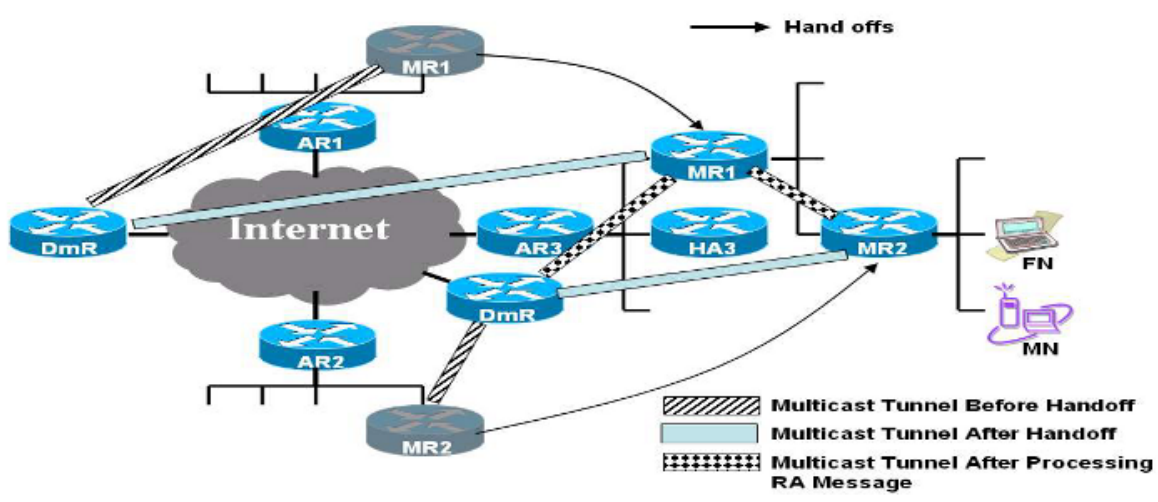

Fig. 1. Operation Flow

When a mobile network acts for the first time, the MR in the network must establish a multicast tunnel with its upper multicast router. To do that, the cases are either the MR find a multicast router by itself, or upper routers(the AR or upper MR) must inform the MR of DmR information. For the former case, if there is no local multicast router, MR cannot find one by itself, and it cannot provide multicast service on its network. For the latter case, if the MR gets DmR information, it will make a multicast tunnel to the DmR and provide successful multicasting. Therefore, in our architecture, we assume that upper routers add DmR information in RA message, and 
a $\mathrm{MR}$ receiving the information must process it. If upper routers send RA message with no DmR information, the MR must understand there is no multicast service under the router and does nothing.

Once a MR gets the information about DmR from RA message, it will establish a tunnel using its new CoA for multicast route tree construction. If the MR is attached under an AR directly, it will receive a RA message with ' $M$ ' bit not checked; then the MR must establish a tunnel to the DmR specified in RA message. On the other hand, if the mobile network is nested under another mobile network, the MR will receive a RA message with ' $M$ ' bit set. In this case the (nested) MR has to make a tunnel to its upper MR, because the DmR information with ' $M$ ' bit set in RA message means that upper MR provides multicast service.

Once the MR establishes a multicast tunnel, it changes old DmR information to newly-acquired one. The new DmR is used for its own RA message, and will be used later when the mobile network moves. We will explain about this later in detail. At this point, all IGMP messages from MNs are handled properly by MR and MNs will receive multicast data.

When a mobile network that has a multicast tunnel moves away, its multicast tunnel using the MR's previous CoA is of no use. Thus, after the MR finishes its registration request process, it has to establish a multicast tunnel to its DmR again using its new CoA and destroy previous one. There is no tree reconstruction cost or delay for this, because the DmR already has provided the multicast group it joined.

After that, the MR starts finding a DmR in the new network by examining RA messages for new DmR information. As stated above, if the MR is attached under an AR, it establishes another multicast tunnel with new DmR; if it is nested it establishes a tunnel with upper MR. Once a new multicast tunnel is established, the previous tunnel with old DmR is destroyed.

Here, we presented a mechanism to provide optimal routing for multicast data in mobile environments. Multicast packets from sender will be routed optimally to the designated MR because of PD mechanism; they will be routed efficiently because of using an appropriate multicast router. The transition is seamless because, when a mobile network changes its location, the MR keeps an old tunnel to the previous DmR until a better tunnel is established. Our approach provides a basic multicast support mechanism on NEMO environments.

\subsection{Extensions}

We extended each part of components in mobile network to support multicasting. Firstly, because the DmR information and ' $M$ ' bit must be specified in RA message, we extended some of the RA message format and stated those below

(1) Default Multicast Router Information Field

- Extended part of Valid Options field to store DmR address information

(2) RA Message Bit, named 'M'

- One of the reserved bits in RA message

- Because the AR may notice the DmR information to its nodes using the RA message, the AR must be extended like below

(1) Default Multicast Router(DmR) Information Configuration

- AR may have DmR information in its configure file 
- AR must add DmR information on DmR field of RA message, only if it has the configure

The most important part of the extension is the functions of the MR, because it is in charge of the mobility. Many parts to extend in our architecture are in it. We stated those below.

(1) Default Multicast Router Information Process

- MR must be able to detect 'M' bit in RA message

- MR must be able to examine DmR Information field in RA message and advertise it on its own network

(2) Multicast Router Functions

(3) Multicast Tunnel Establishment

- MR must be able to establish multicast tunnel with multicast router specified in DmR Information Field

- After handoff, if RA message has ' $M$ ' bit, the MR must re-establish multicast tunnel with its upper MR

- If a MR gets the two multicast tunnels, it must destroy DmR's as soon as it receives multicast data from upper MR

(4) Prefix Delegation Mechanism for Route Optimization

- A MR must set its CoA using PD mechanism when it is nested

\subsection{Characteristics}

As presented in the previous sections, our mechanism eliminates the complexities of multicast data flows on NEMO environments. Specifically, in our method,

A. There is no Pinball Route problem of multicast data

B. There is no bi-directional tunnel for multicast data between MR and its HA

C. Once a MR has provided multicast service in home network, it can permanently provide multicast service till it is shut down

D. MRs in mobile networks have native route path for multicast data

E. Our mechanism minimizes multicast tree reconstruction delay

However, if nested MR supports multicast service and upper MR does not, multicast service is possible for descendants of nested MR.

\section{Performance Evaluation}

We compared cost variables of providing multicast among MIP-BT, MIP-RS, nativerouted multicast, and our mechanism. Parameters needed to compute cost variables are stated below:

L1 : mean time of latency between nested MRs under the designated mobile network

L2 : mean time of latency between a AR and its DmR

L3 : mean time of latency between a AR and all HA that their MR is nested under the AR

L4: mean time of latency between all home networks that one of their MR is nested under the designated AR 
$\mathrm{m}$ : multicast packets between $\mathrm{CN}$ and on-link node in the designated mobile network

$\mathrm{n}$ : $\quad$ nesting level of MR counting from AR

$r$ : total number of nodes joining a multicast group in a designated mobile network

$\mathrm{s}: \quad$ mean time for multicast tree reconstruction

$\alpha: \quad$ cost constant for latency rate under AR's network

$\beta$ : $\quad$ cost constant for latency rate outside of AR's network.

$\omega$ : weight constant for processing a tunnel in a router

$\theta$ : cost constant for multicast tree reconstruction per second

We assumed an environment where a mobile router is moving deeper from root router AR in the designated network. And then, we can write down cost values (1), (2), (3) and (4)

$$
\text { Adopting Native Route Path }=(\alpha \mathrm{L} 2+\beta \mathrm{n} \text { L1 }) \mathrm{m}
$$

MIP-BT on NEMO basic support $=(\alpha \mathrm{L} 4 \mathrm{n}+\mathrm{L} 3+\mathrm{L} 1 \mathrm{n} \alpha \beta+\mathrm{n} \omega) \mathrm{r} \mathrm{m}$

MIP-RS on NEMO basic support $=(\alpha \mathrm{L} 4 \mathrm{n}+\mathrm{L} 3+\mathrm{L} 1 \mathrm{n} \alpha \beta+\mathrm{n} \omega) \mathrm{m}+\theta \mathrm{s}$

$$
\text { our architecture }=(\alpha \mathrm{L} 3+\beta \mathrm{nL} 1) \mathrm{m}
$$

We assume that $\alpha$ is 0.2 and $\beta$ is 2 , because $\alpha$ is the value of the local network and the local network latency may be closer to 0 . And we assume that $\omega$ is 50 and $\mathrm{m}$ is 10 . With these variables, we can draw a graph of fig 2 . Note that (1) cannot occur on NEMO basic support architecture, because of the Pinball Route problem; L1 L4 are generated by supposing that L4 $>$ L3 $>$ L2 >= L1. In Conclusion, Fig. 2 shows our architecture is the most efficient mechanism in multicasting on mobile network.

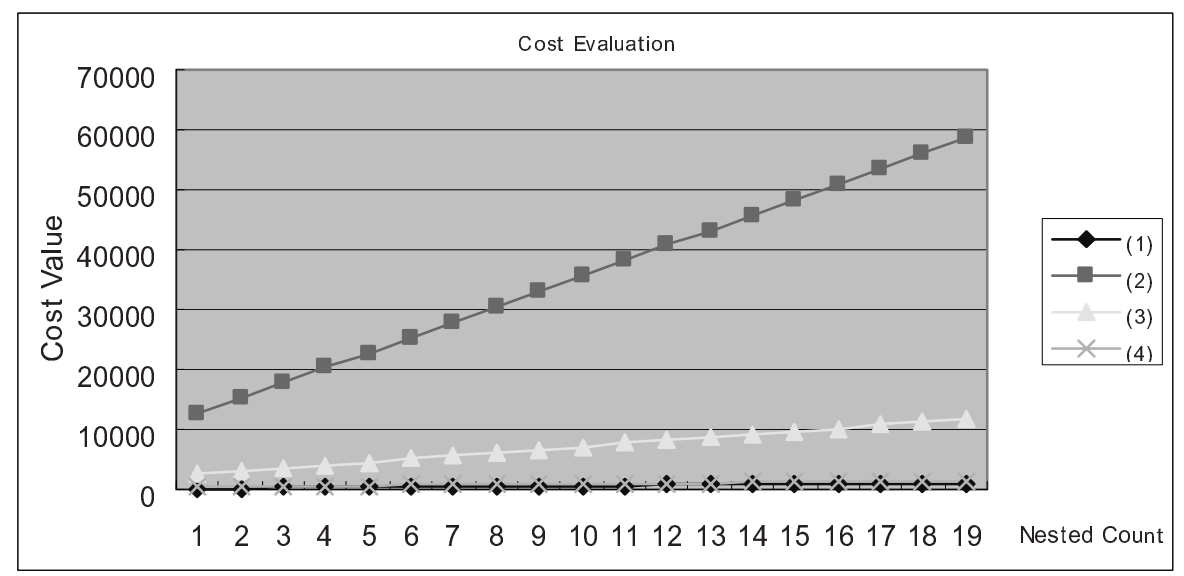

Fig. 2. Cost Evaluation Graph 


\section{Conclusion}

In this paper, we examined and analyzed problems of providing multicast service on NEMO basic support architecture, and proposed new one that provides efficient and optimized route path for multicasting in NEMO. With our architecture, we achieved optimal routing for multicasting by adopting PD mechanism; efficient multicasting by using DmR; and seamless multicasting by keeping an old tunnel to the previous DmR as needed while a mobile network changes its location.

We extended some functions of MR and AR in order to support this. Because this extension is based on the Mobile IP and the IPv6 standards in terms of the functions and protocols, it does not result in much modification or loads to the network components. We also developed a cost analytic model to evaluate the performance of our approach. It showed much lower total cost for on-link nodes in a mobile network, compared to other techniques such as MIP-BT and MIP-RS on NEMO basic support architecture.

Since our approach provides optimal and efficient multicast service and solves the pinball route problem in mobile networks environments, this architecture can become a basic multicast support mechanism on NEMO environments.

\section{References}

1. D. Johnson, C. Perkins, J. Arkko, Mobility Support in IPv6, Internet Draft, <draft-ietfmobileip-ipv6-24.txt>, Jun 2003.

2. Thierry Ernst, Network Mobility Support Goals and Requirements, Internet Draft, <draftietf-nemo-requirements-01.txt>, May 2003.

3. Thierry Earnst, Hong-You Lach, Network Mobility Support Terminology, Internet Draft, < draft-ietf-nemo-terminology-00.txt>, May 2003.

4. Vijay Devarapalli, Ryuji Wakikawa, NEMO Basic Support Protocol, Internet Draft, < draft-ietf-nemo-basic-support-01.txt>, Sep, 2003.

5. Kyeong-Jin Lee, J H Jeong, Route Optimization for Mobile Nodes in Mobile Network based on Prefix Delegation, Internet Draft, <draft-leekj-nemo-ro-pd-00.txt>, Jun 2003.

6. W. Fenne, Internet Group Management Protocol, version 2, RFC2236.

7. V. Chikarmane et al, Multicast Support for Mobile Hosts Using Mobile IP: Design Issues and Proposed Architecture, ACM/Baltzer Mobile Networks and Applications, vol 3, no 4, pp 365-379, January 1999.

8. Hee-Sook Shin, Young-Joo Suh, and Dong-Hee Kwon, Multicast Routing Protocol by Multicast Agent in Mobile Networks, Proceedings of the Proceedings of the 2000 International Conference on Parallel Processing, pp 271-278.

9. Kuang-Hwei Chi, Chien-Chao Tseng and Ting-Lu Huang, A Framework for Mobile Multicast using Dynamic Multicast Route, The computer journal, vol 42, no 6, 1999.

10. Thubert, P., and Molteni, M., Taxonomy of Route Optimization Models in the NEMO Context, Internet Draft: draft-thubert-nemo-ro-taxonomy-00, Oct 2002 